\title{
Intelligent Health Services Based on Biomedical Smart Sensors
}

\author{
Ricardo Colomo-Palacios ${ }^{1}$ (D) Juan A. Gómez-Pulido ${ }^{2, *(D)}$ and Alfredo J. Pérez ${ }^{3}$ (D) \\ 1 Department of Computer Science, Østfold University College, 1783 Halden, Norway; \\ ricardo.colomo-palacios@hiof.no \\ 2 Department of Technologies of Computers and Communications, University of Extremadura, \\ 10003 Cáceres, Spain \\ 3 TSYS School of Computer Science, Columbus State University, Columbus, GA 31907, USA; \\ perez_alfredo@columbusstate.edu \\ * Correspondence: jangomez@unex.es
}

Received: 24 November 2020; Accepted: 25 November 2020; Published: 27 November 2020

\begin{abstract}
Health services can be improved by means of intelligent techniques that handle efficiently massive volumes of data collected from biomedical variables. Nowadays, these services are not only oriented to disease diagnosis and prevention, but wellness too. Advanced technologies and last trends in computing, internet of things, sensors, and data science are driving the development of new systems and applications in the area of intelligent health services based on biomedical smart sensors that deserve to be known. Through five research articles and a review, this Special Issue provides the opportunity to obtain a representative view of the potential of these technologies when applied to such a human welfare-oriented area.
\end{abstract}

Keywords: biomedical sensors; Internet of Things; biomedical data; data mining and big data analysis; intelligent systems for health; machine and deep learning; diagnostic and predictive analytics; healthcare and wellness; activity recognition in health care; data authentication and security

\section{Introduction}

Advances in computer technologies are driving significant changes in medical care by shifting the hospital-centered paradigm to a patient-centered one. The focus on disease is replaced with the orientation to wellness. Monitoring and analyzing biomedical variables are key activities required for diagnosis and health care. The automation of these activities by means of computing systems allows processing massive volumes of data collected from biomedical sensors, leading to useful field applications to health personnel. This is particularly interesting to predict the diagnosis of certain diseases suffered by the most vulnerable groups, like the elderly. New systems, applications, developments, models, and research that make use of monitored medical data are envisaged to bring differentiated services to the society.

Big data and machine learning provide significant potential for this purpose, leading to new applications, more effective operations, and more human approaches. These methodologies enable digging massive databases, enhancing the knowledge base and producing new data model-based applications and services for society. The most significant requirement for these technologies is the availability of databases to mine them or to train and test the models. However, medical information is hard to obtain for administrative issues, so other efficient alternatives to collect data are required. The use of smart sensors requires strategies to minimize interference with the work of health personnel and have a minimal impact on monitored patients. The implementation of health-care services with these technologies will probably save costs, but the benefits will be better perceived as an increment of 
the satisfaction of patients and personnel. Moreover, these technological implementations will expand accessibility to new areas.

This Special Issue provides six manuscripts with original advances in health applications and services propelled by artificial intelligence, big data, and machine learning, supported by the design of biosensor systems for the construction of trustable medical databases. These papers were accepted among sixteen papers submitted to this Special Issue, after a careful review process. All the submissions fitted some of the keywords listed above.

\section{Summary of the Contributions in This Special Issue}

The first article is entitled "Smart Footwear Insole for Recognition of Foot Pronation and Supination Using Neural Networks" [1], by Manuel J. Domínguez-Morales, Francisco Luna-Perejón, Lourdes Miró-Amarante, Mariló Hernández-Velázquez and José L. Sevillano-Ramos. This research focuses on preventing possible pathologies with regard to foot postures, by measuring of foot plantar pressures in both dynamic and static conditions. To this end, the authors have developed a plantar pressure measurement system to identify areas with higher or lower pressure load, which is composed of an embedded system (low-power consumption microcontroller, some force sensor resistances and a low-energy bluetooth module) placed in the insole and a user graphical application. The software receives and shows the insole pressure information in real-time and provides information about the foot posture. The accuracy and usefulness of this information comes from a deep learning neural network system that identifies the different pressure states. This neural network is trained by using a stored dataset in order to obtain the classification results in real-time. As a result, the plantar pressure measurement system provides an accuracy over $90 \%$, considering a training dataset of 3000 steps collected from 6 different users.

The second article is entitled "PISIoT: A Machine Learning and IoT-Based Smart Health Platform for Overweight and Obesity Control" [2], by Isaac Machorro-Cano, Giner Alor-Hernández, Mario Andrés Paredes-Valverde, Uriel Ramos-Deonati, José Luis Sánchez-Cervantes and Lisbeth Rodríguez-Mazahua. The authors discuss the limitations of the Internet of Things (IoT) objects used for collecting data and analyzing health conditions with regard to overweight and obesity, in order to generate recommendations to prevent them. Therefore, the authors propose an alternative based on machine learning to this end. They have developed a machine learning and IoT-based smart health platform for the prevention, detection, treatment, and control of overweight and obesity, and other associated conditions or health problems. The system pursues three main purposes: to identify critical variables, classify patients, and generate medical recommendations. The proposed system was applied to the prevention of myocardial infarction in elderly patients with obesity by monitoring biomedical variables.

The third article is entitled "Evaluating Information-Retrieval Models and Machine-Learning Classifiers for Measuring the Social Perception towards Infectious Diseases" [3], by Oscar ApolinardoArzube, José Antonio García-Díaz, José Medina-Moreira, Harry Luna-Aveiga and Rafael Valencia-García. This research tries to improve the early-detection systems of infectious diseases, specifically in the field of infodemiology, which analyzes online information regarding public health that aims to complement traditional surveillance methods. Infodemiology processes big data by means of techniques for handling natural language efficiently. However, the authors do not find comprehensive studies that compare the accuracy of these techniques. To this end, the authors have conducted an infodemiology-based study to extract positive or negative utterances related to infectious diseases. First, they have used Twitter to compile and label a balanced corpus of infectious diseases, with 6164 utterances written in Spanish and collected from Central America. Next, they compared two statistical-models: word-grams and char-grams. The research focuses on analysing different gram sizes by using two machine learning classifiers: random forest and sequential minimal optimization. The char-grams model with five-char-gram sequences reached an accuracy of $90.80 \%$. 
The fourth article is entitled "Wearable Accelerometer and sEMG-Based Upper Limb BSN for Tele-Rehabilitation" [4], by Ahmed Baraka, Heba Shaban, Mohamad Abou El-Nasr and Omneya Attallah. In this research, the data sources are wearable sensors (particularly, accelerometers) and surface electromyography, and the objective is tele-rehabilitation in human locomotion for people with chronic diseases. The authors propose new positions for the sensors and compare the measured signals to similar existing techniques. For example, the authors found that positioning the surface EMG sensors on the forearm muscles provide more reliable results in the classification of motion abnormality than if they are located in biceps muscles. The statistical and time domain features extracted from accelerometer and EMG signals were used to implement six machine learning classifiers (decision tree, linear discriminant analysis analysis, k-nearest-neighbor, support vector machine, boosted tree and bagged tree) for the automatic classification of Parkinson's tremor. The main result shows that highest classification accuracy $(99.6 \%)$ is provided by bagged tree classifier.

The fifth article is entitled "An Agile Approach to Improve the Usability of a Physical Telerehabilitation Platform" [5], by Hennry Pilco, Sandra Sanchez-Gordon, Tania Calle-Jimenez, Jorge Luis Pérez-Medina, Yves Rybarczyk, Janio Jadán-Guerrero, César Guevara Maldonado and Isabel L. Nunes. This research focuses on performing an usability evaluation of a telerehabilitation platform that combines telecommunication technologies and biomedical smart sensors. The usability was improved by developing an iterative process for a prototype of a telerehabilitation platform, where a digital representation of the patient replicates the therapeutic exercise of the patient, being detected by a Kinect camera and sensors in real time. Furthermore, a cognitive workload assessment was performed to complement the usability evaluation. This proposal allowed one to reduce usability issues cataloged as catastrophic, major, and minor. Finally, the research can be applied as a guide to improve the usability of e-Health systems at the same time that they are programmed.

Finally, this Special Issue includes the review article entitled "Use of Wearable Sensor Technology in Gait, Balance, and Range of Motion Analysis" [6], by Steven Díaz, Jeannie B. Stephenson and Miguel A. Labrador. This paper surveys the current area of wearable sensor technology in gait, balance, and range of motion research from people suffering from neurological disorders. Future research works in this area can take advantage of the current solutions and challenges described in this article. To this end, a two-level taxonomy of rehabilitation assessment is introduced with evaluation metrics and common algorithms utilized in wearable sensor systems.

Funding: This research received no external funding.

Acknowledgments: We would like to acknowledge all of the authors and peer reviewers for their contributions to this Special Issue. We would also like to thank the editorial board of Applied Sciences for trusting our proposal of Special Issue and the editorial team at MDPI for the help in handling the editorial process.

Conflicts of Interest: The authors declare no conflict of interest.

\section{References}

1. Domínguez-Morales, M.J.; Luna-Perejón, F.; Miró-Amarante, L.; Hernández-Velázquez, M.; SevillanoRamos, J.L. Smart Footwear Insole for Recognition of Foot Pronation and Supination Using Neural Networks. Appl. Sci. 2019, 9, 3970. [CrossRef]

2. Machorro-Cano, I.; Alor-Hernández, G.; Paredes-Valverde, M.A.; Ramos-Deonati, U.; Sánchez-Cervantes, J.L.; Rodríguez-Mazahua, L. PISIoT: A Machine Learning and IoT-Based Smart Health Platform for Overweight and Obesity Control. Appl. Sci. 2019, 9, 3037. [CrossRef]

3. Apolinardo-Arzube, O.; García-Díaz, J.A.; Medina-Moreira, J.; Luna-Aveiga, H.; Valencia-García, R. Evaluating Information-Retrieval Models and Machine-Learning Classifiers for Measuring the Social Perception towards Infectious Diseases. Appl. Sci. 2019, 9, 2858. [CrossRef]

4. Baraka, A.; Shaban, H.; Abou El-Nasr, M.; Attallah, O. Wearable Accelerometer and sEMG-Based Upper Limb BSN for Tele-Rehabilitation. Appl. Sci. 2019, 9, 2795. [CrossRef] 
5. Pilco, H.; Sanchez-Gordon, S.; Calle-Jimenez, T.; Pérez-Medina, J.; Rybarczyk, Y.; Jadán-Guerrero, J.; Maldonado, C.; Nunes, I. An Agile Approach to Improve the Usability of a Physical Telerehabilitation Platform. Appl. Sci. 2019, 9, 480. [CrossRef]

6. Díaz, S.; Stephenson, J.B.; Labrador, M.A. Use of Wearable Sensor Technology in Gait, Balance, and Range of Motion Analysis. Appl. Sci. 2019, 10, 234. [CrossRef]

Publisher's Note: MDPI stays neutral with regard to jurisdictional claims in published maps and institutional affiliations.

(c) 2020 by the authors. Licensee MDPI, Basel, Switzerland. This article is an open access article distributed under the terms and conditions of the Creative Commons Attribution (CC BY) license (http:/ / creativecommons.org/licenses/by/4.0/). 